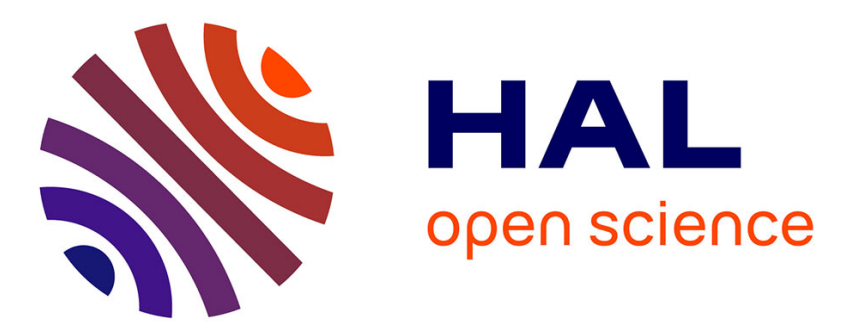

\title{
Variational segmentation of textile composite preforms from X-ray computed tomography
}

Jean Bénézech, Guillaume Couégnat

\section{To cite this version:}

Jean Bénézech, Guillaume Couégnat. Variational segmentation of textile composite preforms from X-ray computed tomography. Composite Structures, 2019, 230, pp.111496. 10.1016/j.compstruct.2019.111496 . hal-02328619

\section{HAL Id: hal-02328619 \\ https://hal.science/hal-02328619}

Submitted on 23 Oct 2019

HAL is a multi-disciplinary open access archive for the deposit and dissemination of scientific research documents, whether they are published or not. The documents may come from teaching and research institutions in France or abroad, or from public or private research centers.
L'archive ouverte pluridisciplinaire $\mathbf{H A L}$, est destinée au dépôt et à la diffusion de documents scientifiques de niveau recherche, publiés ou non, émanant des établissements d'enseignement et de recherche français ou étrangers, des laboratoires publics ou privés. 


\title{
Variational segmentation of textile composite preforms from X-ray computed tomography
}

\author{
Jean Bénézech ${ }^{\mathrm{a}, \mathrm{b}}$, Guillaume Couégnat ${ }^{\mathrm{a}, *}$ \\ ${ }^{a}$ Laboratoire des Composites Thermostructuraux, UMR5801 CNRS/Univ. Bordeaux/Safran/CEA, \\ 3 allée de la Boétie, 33600 Pessac, France \\ ${ }^{b}$ Safran Ceramics, 105 avenue Marcel Dassault, 33700 Mérignac, France
}

\begin{abstract}
Prediction of the thermo-mechanical behavior of woven composites necessitates a reliable knowledge of their inner structure. A sufficiently accurate description of the fabric geometry could be obtained using X-ray computed microtomography $(\mu \mathrm{CT})$ at the mesoscopic scale. However, systematic construction of numerical models from $\mu \mathrm{CT}$ remains a difficult task. To address this challenge, we propose a variational segmentation approach which combines $\mu \mathrm{CT}$ with a prior geometric model that is iteratively improved thanks to a heuristic optimization process. The fidelity of the models with respect to the input $\mu \mathrm{CT}$ is evaluated using a measure of similarity including both gray levels and local directions. Our method allowed to build realistic numerical models of woven fabrics that preserve the prescribed weaving pattern, and that are free of interpenetration, which makes them compatible with further numerical simulations. Using our approach, models of complex woven fabrics, but also of woven composites, could be consistently generated from $\mu \mathrm{CT}$ and can serve as reference models, e.g. to analyze in situ tests by pro-
\end{abstract}

\footnotetext{
*Corresponding author

Email addresses: benezech@lcts.u-bordeaux.fr (Jean Bénézech), couegnat@lcts.u-bordeaux.fr (Guillaume Couégnat)
} 
viding numerical twins.

Keywords: woven composites, textile, X-ray computed tomography, meso-scale

\section{Introduction}

There is a growing interest in the use of textile reinforcement for advanced composite applications: woven fabric composites offer a large improvement in manufacturability, through-thickness mechanical properties and damage impact resistance over their laminate counterparts [1]. However, because of their intricate architecture structure, the geometry of the reinforcement has a strong influence on the mechanical properties of woven composites and must be taken into account.

The mesoscopic scale has emerged as the most relevant scale to include the architectural details of the textile preform, and to simulate the behavior of woven composites [2, 3]. It offers a good compromise between the description of the material internal details while keeping the computational costs acceptable. However, the creation of representative numerical models at this scale remains challenging: it supposes to be able to provide a reliable description of the geometry of the tows and to generate a quality finite element mesh from this description [4, 5]. Various modeling approaches have been proposed in the literature [6], which differ in the level of detail incorporated in the description of the reinforcement geometry.

Geometric approaches rely on an a priori knowledge of the weaving pattern and idealize the shape of the tows [7, 8]. These approaches require minimal computational effort but typically lead to unrealistic yarn cross sections for complex fabrics. Improvements could be achieved by locally modifying the shape of the yarns based on observations on real fabrics [9, 10]. This step could be automatized by adding a relaxation step: either by the application of the principle of 


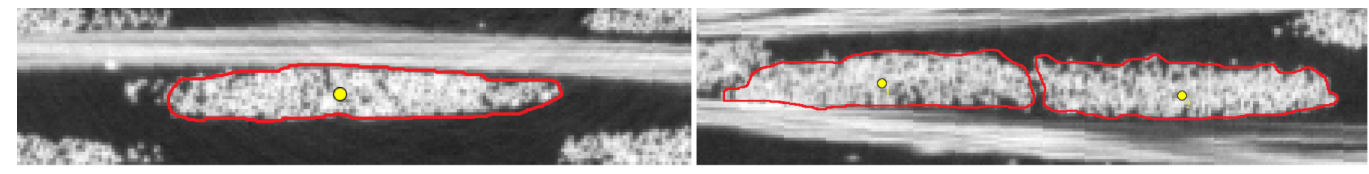

Figure 1: Illustration of the difficulty to define a definitive yarn contour: for a yarn (left), it depends whether single fibers are included within the envelope; for merged yarns (right), the separation is somewhat arbitrary.

minimum energy to the yarn paths [7], or by simulating the compaction of an initial model [11]. More involved approaches use a discrete representation of the yarns [12-16] where the yarns are described as a set of macro-fibers and simulate the relaxation of the preform by explicitly considering the contacts between these macro-fibers. These methods partially alleviate the limitations of the geometric models and allow to obtain yarn shapes closer to the reality of the material [17, 18]. Yet, they remain inadequate to incorporate the variability of complex woven architectures; they are still initially based on an idealized description of the weaving pattern. Stochastic framework have been proposed to include the spatial variability of the yarns paths into geometric models [19-21], but these approaches still need to be calibrated against a large amount of micrographic or micro-tomographic data. One of the main shortcomings shared by all these virtual approaches is the handling of the interpenetration between yarns. Depending on the assumptions for the yarns geometry, these models could lead to inconsistencies which could eventually impede the latter finite element (FE) mesh generation. It requires extra work to correct these interpenetrations: geometric models must be post-processed before they can be meshed [11, 16, 19, 22].

An alternative approach to the generation of virtual models is to reconstruct the textile preform from images of existing material, especially using 3D X-ray 
computed tomography $(\mu \mathrm{CT})$. It allows to embrace the whole complexity of the preform at once and naturally capture the variability of the architecture. Yet, the difficulty of this approach is then to be able to label each yarns. The segmentation process could be done manually for simple 2D preform [20, 23], but it would require excessive manual intervention to be extended to more complex 3D architectures. Several attempts have been made to automatize the segmentation process. Notably, the structure tensor of the image [24, 25] could be use to estimate the local orientation of the yarns and to separate weft from warp. For simple fabrics, it is sufficient to exploit this information alone [26], or combined with other statistical descriptors [27, 28], to extract each yarn individually. However, for more complex cases, individual yarns still have to be separated and labeled: a sole use of image-based information leads to topological inconsistencies [29]. This is for example the case when two yarns of the same direction merge into a single entity (see e.g. Fig. 1), or when the structure tensor could not be calculated accurately. To disambiguate these cases, it is therefore necessary to reintroduce $a$ posteriori an approximate knowledge about the size of the yarn sections or about the weaving pattern. Yet, heuristics developed to arbitrarily separate the connected entities will not necessarily ensure to obtain a topologically correct model, even using ad hoc approaches (see e.g. [29]). This caveat illustrates the difficulty to conciliate image-based data with a continuous description of the yarns geometry. A promising approach was recently proposed by Mazars et al. [30] to overcome this obstacle: it consists in combining a priori geometric informations within an image-based approach. In a first step, a limited collection of yarn cross-sections is contoured manually. These sections are then propagated using the gray level and the local direction of the yarns. This approach extends the pursuit algorithm pro- 
posed by [29] by introducing an initial manual step that guarantees a topologically correct model.

A fully automatized image-based approach appears yet to be impracticable for complex fabrics: it still needs to be guided by at least some prior knowledge of the weaving pattern and by geometric assumptions. Our objective is to propose an image-based approach to construct realistic and topologically correct mesoscopic models of woven fabrics. The main goal is to improve the robustness of existing image-based methods by providing sufficient a priori information to prevent any topological inconsistencies. We aim at combining the benefits of both geometric approaches, i.e a smooth and continuous description of yarns with a prescribed weaving pattern, and image-based approaches, i.e. a more realistic description of the cross-sections and of the fabric variability.

The principle of our approach and the required input data are first presented in Sec. 2. Implementation details and the optimization framework will then be discussed. The convergence and the sensitivity of our approach will be analysed using a simple example. Finally, our method is compared to other approaches and its capabilities are illustrated with supplementary examples.

\section{Variational segmentation: principle and input data}

\subsection{Principle}

To ally the advantages of geometric and image-based approaches, we propose a variational segmentation method based on the combined use of a prior geometric model and data retrieved from $\mu \mathrm{CT}$ images. The main idea here is to use an initial geometric model, possibly coarse, as a starting point to be optimized with respect to some parameters describing the yarns shape and position. The use of an ini- 
tial geometric model will guarantee that the final segmentation contains the right number of entities (i.e. yarns) and matches the prescribed weaving pattern. The parameters describing the yarns geometry, i.e. their centroid position and their cross-section, will be perturbated to optimize the resemblance of the geometric model with respect to data derived from $\mu \mathrm{CT}$. The resemblance between the improved geometric model and the $\mu \mathrm{CT}$ is quantified using a similarity measure $E$ defined as:

$$
E=\alpha E_{g}+\beta E_{d}-\gamma E_{r}
$$

where $E_{g}$ measures the resemblance in term of gray level between the geometric model and the $\mu \mathrm{CT}$ : it will ensure the envelope of the geometric model will match the mask of the preform, i.e. the yarns will be located only where the $\mu \mathrm{CT}$ gray level corresponds to the textile preform; $E_{d}$ quantifies the consistency between the local directions retrieved from the $\mu \mathrm{CT}$ and the ones from the geometric model: this will help to effectively separate the weft from the warp; the last term $E_{r}$ acts as a regularization term: interpenetration between yarns will be penalized, as well as unrealistic configurations, e.g. exaggerated yarn size. The three components of $E$ could be weighted depending, for instance, on the quality of the input data. Appropriate values of $\alpha, \beta$, and $\gamma$ could be determined to ensure optimal convergence of the algorithm, as discussed in Section 5.1 .

The necessary ingredients to compute the similarity measure, i.e. the prior geometric model, and the information derived from $\mu \mathrm{CT}$ are illustrated in Fig. 2 and are further discussed below. The exact computation of $E_{g}, E_{d}$ and $E_{r}$ are detailed in Sec. 3 

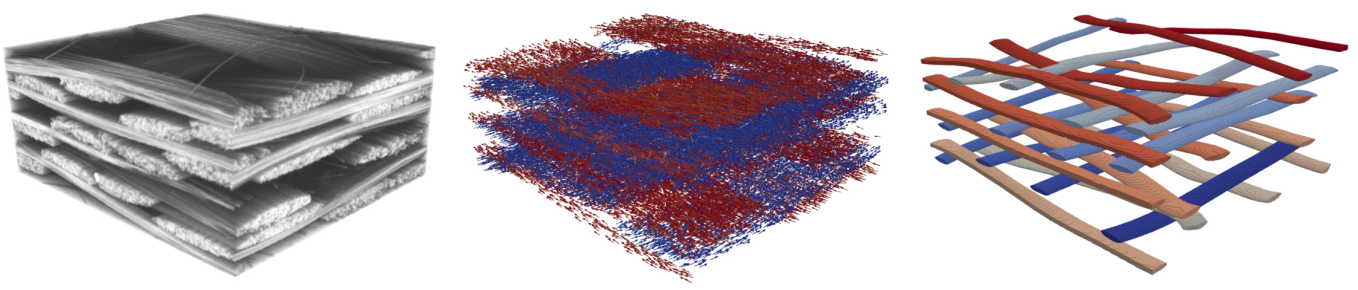

Figure 2: Input data required for our approach: $\mu \mathrm{CT}$ used to define the binary mask of preform (left), local directions of the yarns calculated using the structure tensor of the image (center), and prior geometric model of the fabric (right).

\subsection{Prior geometric model}

The weave geometry is described as a set of yarns. Each yarn is represented by its center path and by a finite collection of cross-sections along this path. The cross-sections are defined in a two-dimensional plane orthogonal to the local tangent of the center path. They are positioned at discrete points along the path.

5 The discretization of the path should be sufficient to allow a smooth description of cross-sections changes. It could be for example chosen as a function of the local curvature. Depending on the shape of the yarns, the cross-sections could be described by ellipses or by polygons to be able to describe non-convex shapes for instance (cf. Fig. 3).

For simple weaves, realistic paths could be directly derived from the weaving pattern using geometric approaches. In the case of more complex patterns, it is still possible to retrieve approximate description of the central paths (see e.g. [31]? ]), however it is much more involved. Yet, idealized description of the middle lines could significantly deviate from the reality [32]: yarns could be shifted from their theoretical position, or some yarns could even be missing. These discrepancies could potentially hinder the relaxation process. In this work, we chose to 

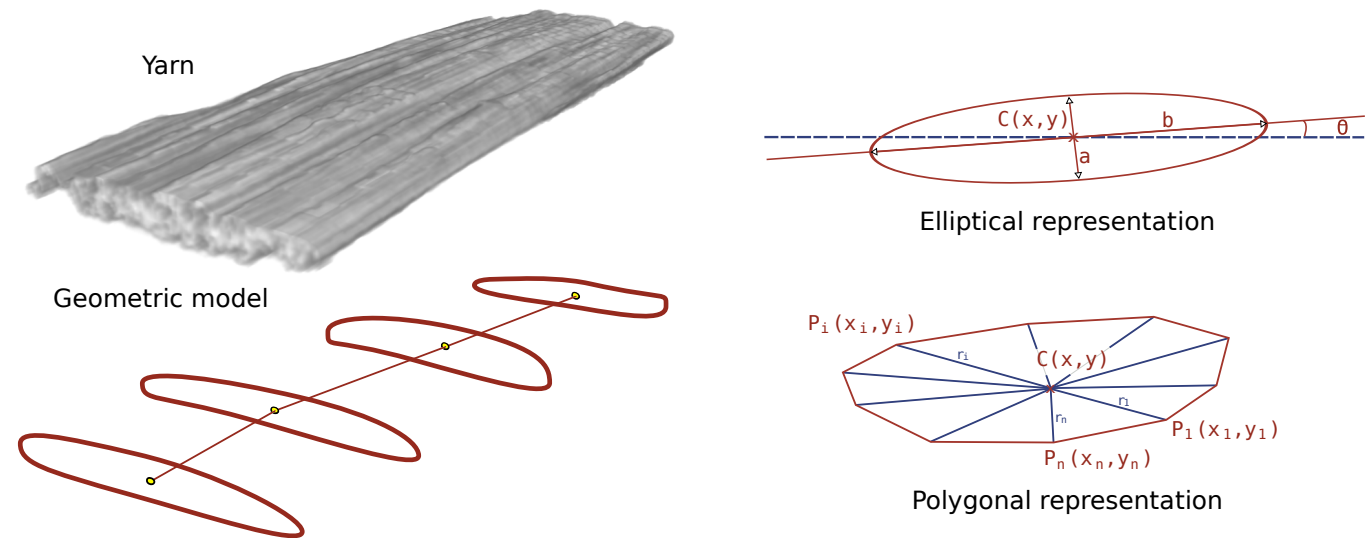

Polygonal representation

Figure 3: Geometric description of a yarn: each yarn is described by a center path and a collection of cross-sections along this path (bottom left). The parameters of each cross-section depend on the chosen geometric representation, i.e. elliptic or polygonal (right).

overcome this potential problem by retrieving the paths directly from $\mu \mathrm{CT}$ of the materials. Some points were manually selected along the paths for each yarn using Fiji [33]. This preprocessing step was sped up by using dedicated macros and by keeping the number of points minimal, only to ensure a correct initial topology. The paths were then interpolated using B-splines to recover a continuous curve.

5 The number of cross-sections along the paths should be sufficient to accurately describe possible section variations. Concerning the size of the initial cross-sections, this choice is not crucial: as it will be shown in Sec. 5.1, the correct size of the yarns is quickly recovered within the first iterations. In the following, we initialize our geometric models with small elliptic cross-sections.

\subsection{Binary mask of the preform}

The grayscale levels in the $\mu \mathrm{CT}$ images, as illustrated on the left side of Fig. 2 , represent the local density of matter. The corresponding histogram is shown in 


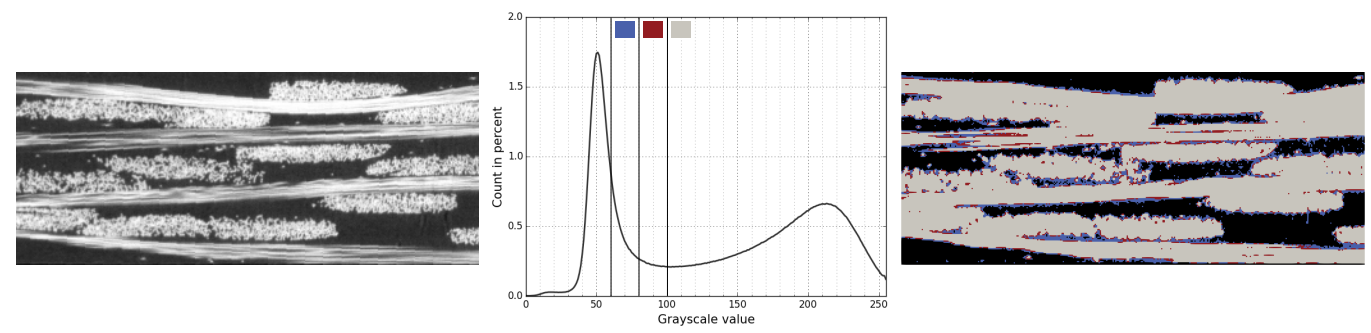

Figure 4: A slice of the $\mu \mathrm{CT}$ scan (left) with its corresponding gray level histogram (center). Resulting binary masks of the preform for three threshold values (right).

Fig. 2.3 and appears almost bimodal: a simple threshold could be used to the separate the yarns from the void. Binary masks obtained for three threshold values - namely 60, 80 and 100 over an 8-bit dynamic - are depicted in Fig. 2.3. For the lower value, background noise and $\mu \mathrm{CT}$ artifacts are still present within the mask, which would be detrimental to the relaxation process. For threshold larger than 5 80, the envelope of the yarns is well captured and free of artifacts. The outline of the resulting mask is quite similar and should not influence the final result of relaxation. The only visible difference is that the higher the threshold, the more the intra-yarn porosity is segmented. To cope with the continuous nature of the yarns in the geometric model, it is wiser to choose a moderate value of the threshold. For this particular $\mu \mathrm{CT}$, a threshold of 80 was selected.

\subsection{Local directions}

The second information which is derived from the $\mu \mathrm{CT}$ is the local directions of the yarns. It is classically computed using the structure tensor $G$ [24, 25] defined as:

$$
G=\nabla I \cdot \nabla I^{T}
$$


where $\nabla I$ is the grayscale gradient obtained with an optimal kernel for fiber materials [34]. The local values of $G$ are convoluted with a Gaussian kernel $K_{\Omega}$ over a neighborhood $\Omega$ to make it more robust against noise.

$$
G_{\Omega}=K_{\Omega} * G
$$

For a given voxel, the local direction of the yarn corresponds to the direction of the eigenvector of $G$ with the smallest eigenvalue. An example of local directions is depicted in Fig. 2 where vectors are colored depending on their orientation in the $x y$ plane. It clearly shows that this direction calculation allows to separate the weft from the warp. Convolution of $G_{\Omega}$ over a larger domain $\Omega$ will certainly improve the quality of the calculation inside the yarns. However, using a domain too large will eventually lead to inaccurate estimations of the local direction between yarns, as the Gaussian kernel will act as a diffusion kernel. In the following, we use a Gaussian kernel smaller than half the size of the yarns thickness.

\section{Computation of the similarity measure $E$}

To assess the resemblance of the geometric model, we need to calculate its similarity $E$ (cf. Eq. 1) with respect to the $\mu \mathrm{CT}$. As the binary mask of the preform and the local directions are naturally available as 3D images since they are derived from the $\mu \mathrm{CT}$, this comparison would be facilitated if the geometric model was also available as an image: it would allow to evaluate $E$ discretely voxel by voxel. Therefore, a prior step before effectively compute the similarity is that the geometric model needs to be voxelized. 

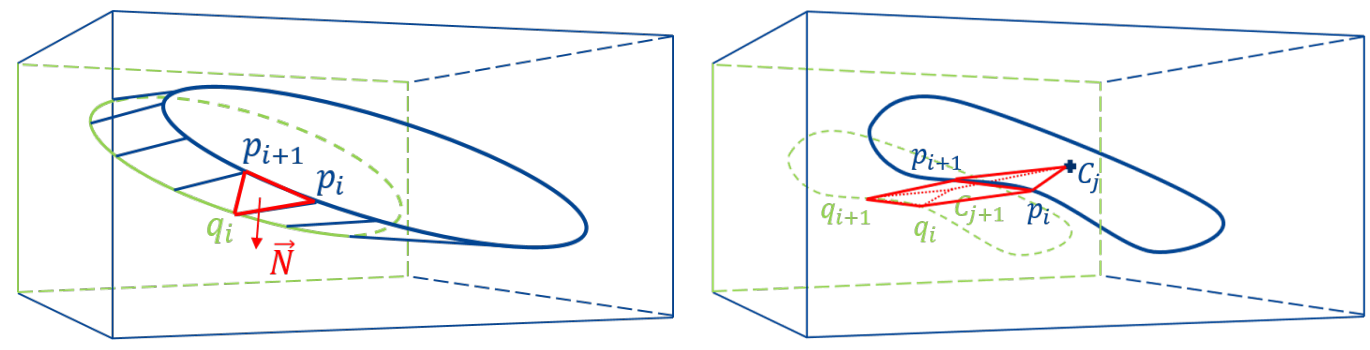

Figure 5: Illustration of the geometric predicates required to perform the voxelization of yarns segments: elliptic (left) and polygonal (right) cases.

\subsection{Voxelization of the geometric model}

Each yarn is divided into segments, i.e. the intervals between two consecutive points along the centerline. The corresponding cross-section boundaries are resampled with a fixed number of points ( $p_{i}$ and $q_{i}$ in Fig. 3.1). This allows to tessellate the outer boundary of each segment as an oriented surface formed by the

5 facets $\left(p_{i}, p_{i+1}, q_{i}\right)$. For a given image resolution, the voxelization simply consists in determining if a voxel is inside or outside a particular segment of a yarn. In the case of elliptic cross-sections, one only needs to check if a voxel is located on the positive or negative half-space (with respect to the facet normal $\vec{N}$, cf. Fig 3.1) of each facet of a segment. If the voxel is on the negative side for every facet of the envelope, it is inside the corresponding segment. For polygonal cross-section, the geometric predicate is a little bit more involved as the envelope could be nonconvex. In this case, one needs to check whether a voxel is inside one of the prism $\left(c_{j}, p_{i}, p_{i+1}, c_{j+1}, q_{i}, q_{i+1}\right)$ forming the segment.

\subsection{Grayscale similarity $E_{g}$}

15 The first term $E_{g}$ measures the similarity between the binary mask of the preform and the voxelized geometric model. For a given subdomain $V$, we determine 
the number $N_{m}$ of non-matching voxels, i.e. voxels that belong to the binary mask but not to the voxelized model, or voxels that belong to the complementary of the binary mask and to the voxelized model. $E_{g}$ is then defined as:

$$
E_{g}=1-\frac{N_{m}}{|V|}
$$

where $|V|$ is the number of voxels of $V$. It is worth noting that this definition is conceptually similar to the Jaccard index or other overlap based metrics [35].

\section{3.3. Direction similarity $E_{d}$}

The second term $E_{d}$ measures the consistency between the local directions of the yarns calculated using the structure tensor of the $\mu \mathrm{CT}$ and the direction of each segment of the geometric model. For each voxel belonging to a yarn in the geometric model, its centroid is projected onto the corresponding centerline (as discussed in [36]); the local tangent at the projected vertex is then defined as the local direction of the voxel. For these voxels, we compare their direction with the ones determined from the $\mu \mathrm{CT}$. If the solid angle between these two vectors is less than a certain value $\left(\theta=15^{\circ}\right.$ in the following), we consider that the directions

agree. $E_{d}$ is finally defined as the ratio between the number of voxels fulfilling this condition and the total number of voxels belonging to the geometric model.

\subsection{Regularization and handling of interpenetrations}

The benefit of the last term $E_{r}$ is twofold: it acts as a regularization term and helps to avoid interpenetrations between yarns in the final segmentation and will be defined as the sum of two contribution $E_{r}=E_{p}+E_{i}$. First, the similarity score is penalized if some geometric criteria are violated, e.g. if the length of the ellipse axis is outside a prescribed range, or if the aspect ratio of a cross-section is above 
a certain limit. These criteria are imposed as hard constraints by setting $E_{p}$ to an arbitrary large penalty value. The second utility of $E_{r}$ is to reduce the presence of interpenetrations between yarns. During the voxelization of the geometric model, each yarn is labeled individually. If two yarns are interpenetrated, a voxel could be attributed multiple labels. An interpenetration ratio is defined as the ratio be5 tween the number of voxels belonging to multiple labels and the size of the current subdomain. $E_{i}$ is directly given by this interpenetration ratio.

\section{Optimization framework}

The input data and the calculation of the similarity have been developed in the previous sections. We can now look towards how to optimize the prior geometric model by increasing its resemblance with the $\mu \mathrm{CT}$. As already discussed above, the main idea is to perturbate the parameters describing the geometry of the yarns, i.e. the position and the shape of the cross-sections to improve the resemblance of the model. The number of parameters could grow rapidly, from a few 1,000 for small models to over 100,000 for larger ones. Therefore, a global optimization approach seems unrealistic. Moreover, the objective function is not guaranteed to be smooth nor convex, and will probably exhibit multiple local extrema: a gradientbased approach is not expected to be feasible. To overcome these difficulties, we propose an optimization framework based on an iterative global-local approach driven by a heuristic algorithm.

\subsection{Iterative global-local approach}

The perturbation of a parameter will cause local modifications of the geometric model, but only over a limited domain, as each cross-section is shared by only two segments. Thus, modifications of cross-sections that are separated by a few 


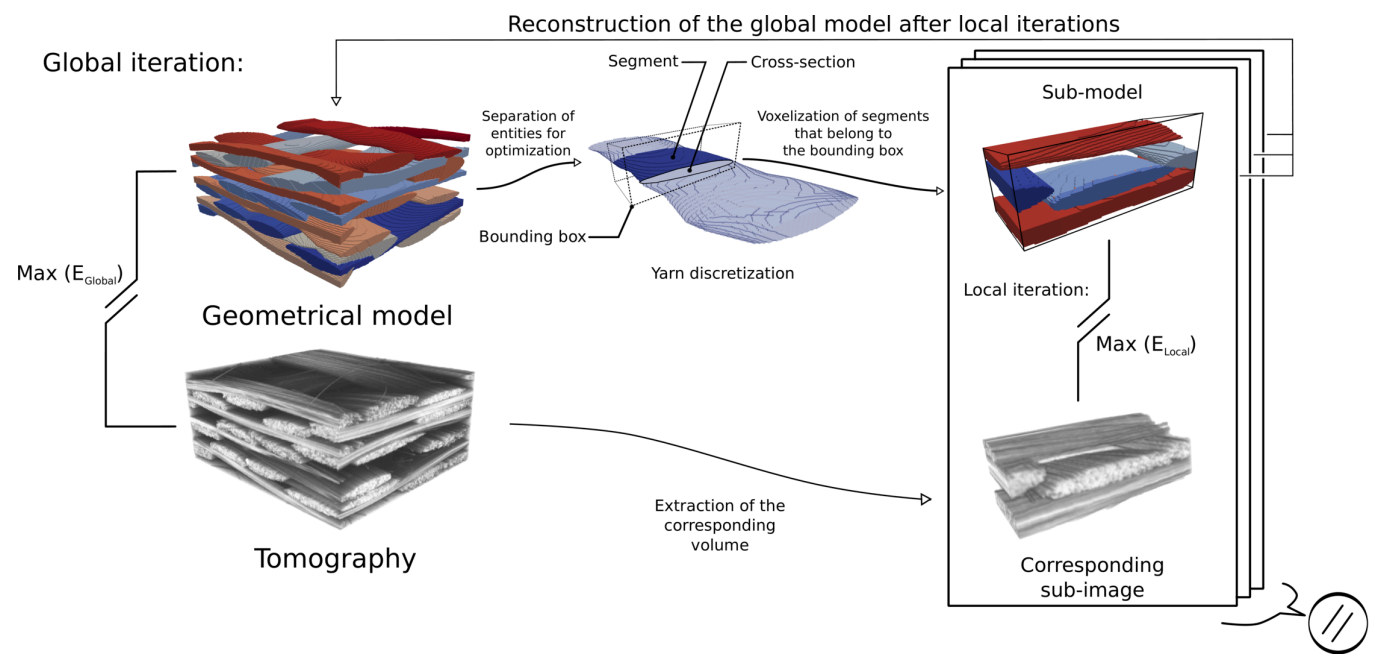

Figure 6: Schematic of the iterative global-local approach.

segments are independent. Then, for each segment, we consider a local sub-model delimited by its bounding box enlarged by a sufficient size (cf. Fig 4 , top right): this extra buffer ensures that any geometric modification will be contained within the sub-model. As the sub-models are constructed independently, they can be easily processed in parallel. Yet, as a cross-section is shared by two consecutive segments, it will be modified twice, i.e. in both segments sub-models. Race conditions could also occur if consecutive segments are processed simultaneously. Thus, the process order of the sub-models is shuffled at each global iteration to both minimize race conditions, and to avoid a geometric bias caused by a systematic evaluation order. When every sub-model has been processed, the global geometric model is updated and a new collection of sub-models is built, as illustrated in Fig 4 . This procedure is iterated until a stationnary point is reached. The convergence is monitored by following the evolution of both the global similarity $E$, and each of its components $\left(E_{g}, E_{d}\right.$ and $\left.E_{r}\right)$. 


\subsection{Local heuristic optimization}

Each sub-model is constructed around a segment, i.e. two cross-sections are involved. For each cross-section, the parameters to be optimized are the position of the centroid, for both elliptic and polygonal sections; for elliptic cross-sections the other parameters are the semi-axis $a$ and $b$, and the orientation $\theta$ (cf. Fig 3);

5 for polygonal sections the vertices $p_{i}$ of the contour are parametrized by their distance $r_{i}$ from the centroid (cf. Fig 3). In total, for each cross-section, there are 5 parameters for the elliptic case and $n+2$ for a polygonal one, where $n$ is the number of vertices on the contour. Therefore, for each sub-model there are twice this number of parameters to be optimized. Local optimization of the sub-models are performed using a heuristic algorithm similar to a Monte-Carlo one. Every parameter of the sub-model is examined sequentially, but in a random order. A trial value is drawn from a uniform distribution in a prescribed range centered around its current value. To assess the influence of this perturbation, the current submodel, i.e. the segment and its neighborhood, needs to be re-voxelized. The trial similarity is then recalculated. If the similarity increases, the value of parameter is updated, otherwise, it is reverted to its previous value. This procedure is then performed iteratively. Here, we do not attempt to reach an optimum during each local optimization: only a few cycles are performed, typically 3-10. Practically, this suffices to ensure a sensible improvement of the similarity without causing extreme geometric variations which could be detrimental for other sub-models.

\section{Results and discussion}

All the procedures discussed above, i.e. the voxelization of the geometric model and of the sub-models, the computation of the similarity, and the pro- 

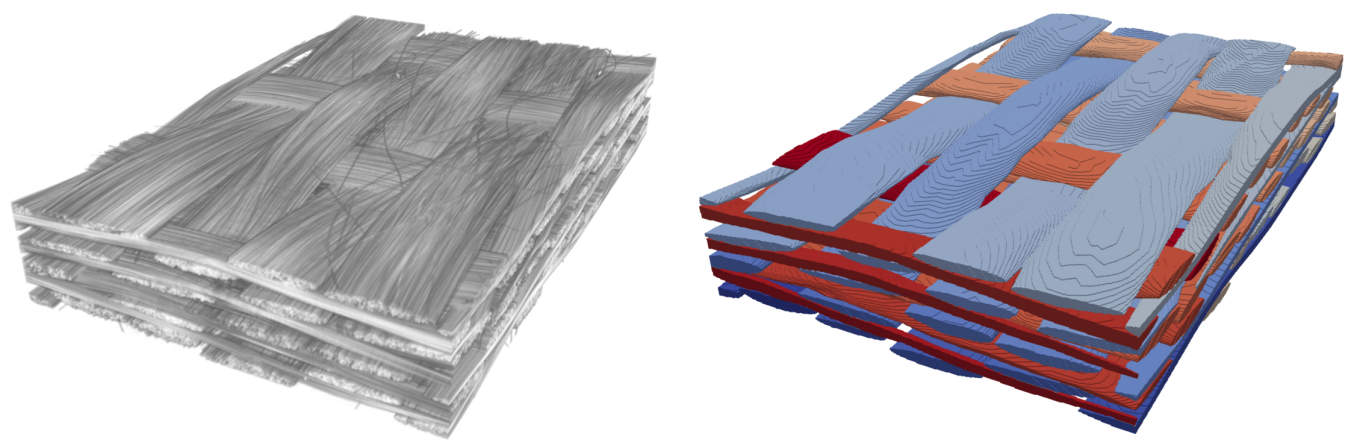

Figure 7: Typical result obtained using our segmentation approach: $\mu \mathrm{CT}$ of a multilayer woven fabric (left) and corresponding optimized model (right).

posed optimization framework, have been implemented into an in-house code using $\mathrm{C}++$. Parallelization of the code has been managed using OpenMP directives. A typical result of our variational segmentation method is illustrated for a multilayer fabric in Fig. 5. With respect to the original $\mu \mathrm{CT}$ of the preform, the comparison is visually satisfying. For reference purpose, this computation ran in 5 less than 3 hours on a 6-core Xeon ${ }^{\circledR}$ E2563 workstation.

This example will serve to analyze the convergence of our procedure, and to provide a better insight on the role of each term of the similarity measure. The sensitivity of our approach with respect to the quality of the input data, especially the resolution of the $\mu \mathrm{CT}$, is investigated. Then, we use the same similarity measure to compare quantitatively our results with segmentations obtained using more classical procedures. Finally, the capabilities of our approach is illustrated through some supplementary examples of increasing complexity. 


\subsection{Convergence analysis}

The convergence of the algorithm mainly depends upon two factors: the shape of the cross-sections (elliptic or polygonal), and the weights of the terms of $E$. Empirically, we obtained the most efficient convergence by first using elliptical cross-sections before switching to polygonal ones, and by adapting the values for $\alpha, \beta$ and $\gamma$ during the global iterations.

The type of cross-section will evidently influence the final geometry of the model, but it will also significantly affect the time to obtain this result. Polygonal cross-sections allow to retrieve more complex shapes, as non-convex ones, yet they necessitate to optimize a larger number of parameters: a global iteration using polygonal cross-sections will necessitate 3 to $5 \times$ more CPU time than for elliptical ones. Practically, we rely on elliptic cross-sections for the first global iterations (up to iteration 50, cf. Fig. 9) as it allows to readily obtain a approximate description of the yarns. Then, for the remaining iterations, we switch to a polygonal description to refine the envelope. Elliptical cross-sections could be viewed as a pre-processing step to the polygonal ones. Even if it was not explicitly tested, the same result should be obtained by using only polygonal cross-sections except it will lead to longer CPU time. We could also anticipate problematic configurations during the first iterations, as it is more involved to prescribe an acceptable aspect ratio to yarns with polygons than with ellipses.

During the first iterations, we emphasize the importance of $E_{g}$ over the other terms $E_{d}$ and $E_{r}$ by initially setting $\alpha=1$ and $\beta=0$ in Eq. 1 ( $\gamma$ is initially set to a small value, $\gamma \ll 1$, so that $\gamma E_{i}$ is negligible with respect to the others terms, i.e. interpenetration is not penalized). Using elliptical cross-sections, it allows to almost instantly retrieve the correct size of the yarns as illustrated on Fig. 8 


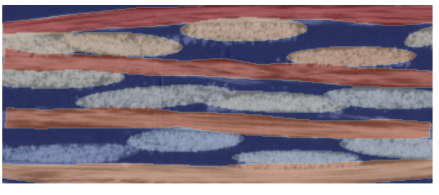

Iteration 10

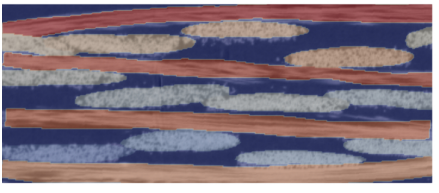

Iteration 62

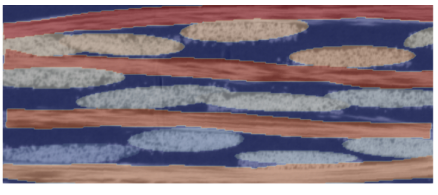

Iteration 37

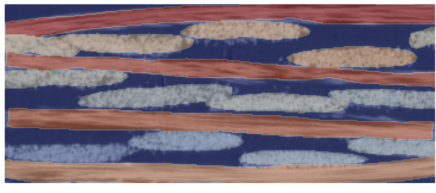

Iteration 80

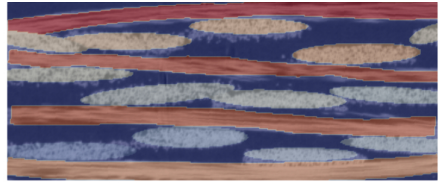

Iteration 50

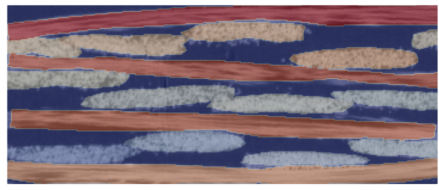

Iteration 150

Figure 8: Evolution of the yarns geometry during the optimization process for the model of Fig.5 For selected iterations, the yarns are colored over a slice of the input $\mu \mathrm{CT}$.

(iteration 10) and on Fig.9 9 where the maximal value of $E_{g}$ is reached only after a few iterations. If the resemblance with the binary mask is almost optimal, weft and warp could still be wrongly assigned and significant interpenetration is evidenced (see $E_{i}$ in Fig. 9). Therefore, during the subsequent iterations, we progressively increment the value of $\beta$ up to a prescribed value (here, $\beta=0.5$ ) while keeping ${ }_{5} \alpha+\beta=1$. This will allow to separate effectively the weft from the warp. As shown in Fig. 9, the value of $E_{d}$ increases linearly up to iteration 37, while the value of $E_{g}$ remains almost constant. To correct the remaining interpenetration, we finally set $\gamma \approx 10$ so that $\gamma E_{i} \approx \alpha E_{g} \approx \beta E_{d}$, i.e. all three terms contribute equally to $E$. As a consequence, $E_{i}$ decreases significantly between iterations 37 and 50. The diminution of the interpenetration is also clearly visible on Fig. 8 (iteration 50) where the yarns are now well separated. A simultaneous decrease of $E_{g}$ is also evidenced, which is related to the use of elliptic cross-sections: as they are not appropriate to capture the real shape of the yarns, the only way to avoid interpenetration is by reducing the size of their cross-section. The result obtained 


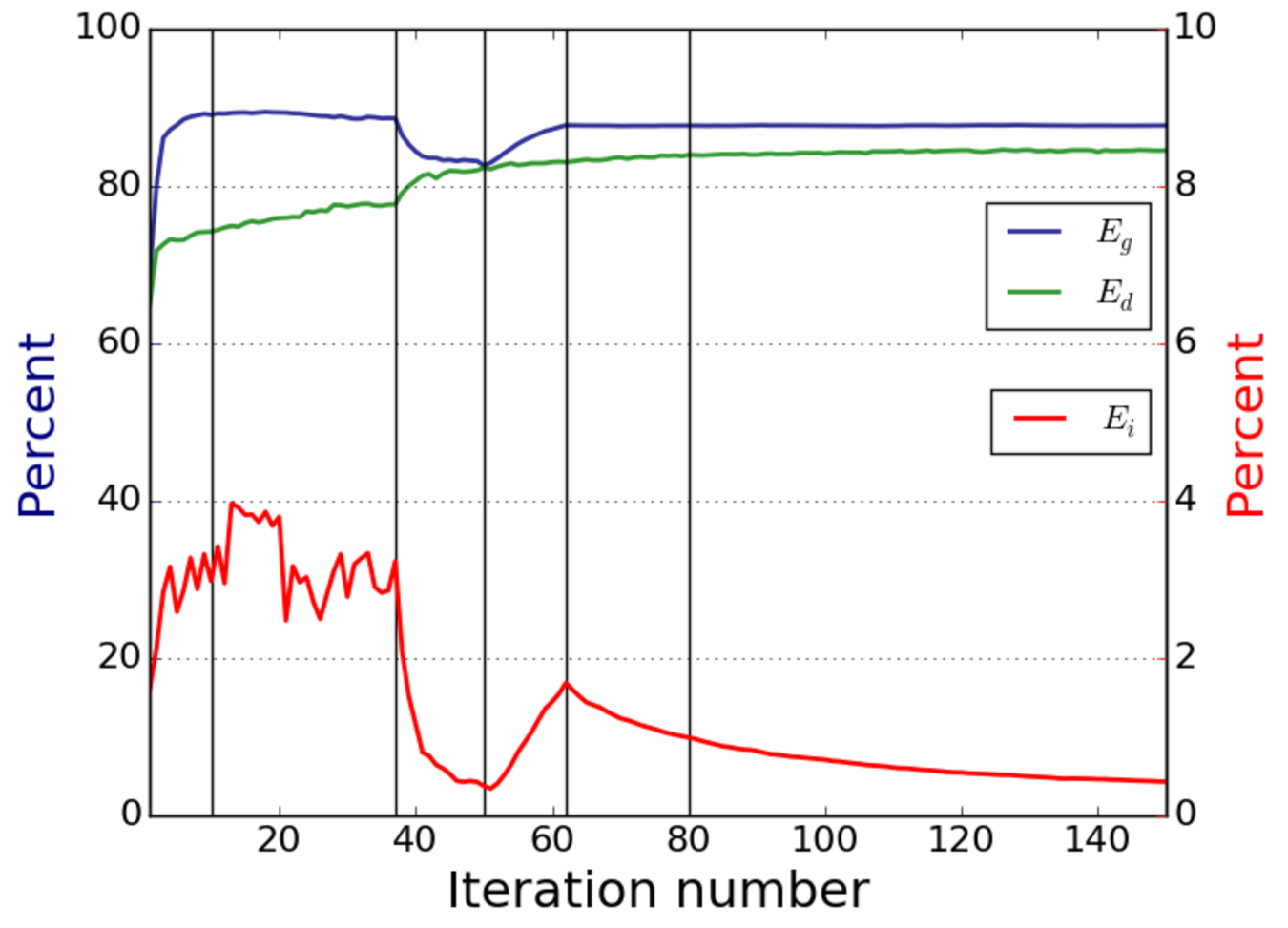

Figure 9: Evolution of the components of the similarity measure $E$ during the optimization. The vertical lines correspond to the snapshots depicted in Fig. 8 
at iteration 50 represents the optimal model we could achieved by using only elliptic cross-sections and by minimizing the interpenetration ratio. As discussed above, after iteration 50, we switch to polygonal cross-sections to further improve the resemblance of the model. To speed up the process, we temporarily release the interpenetration by letting $\gamma \ll 1$. This allows to better fit the envelope of the

5 yarns (see Fig. 8, iteration 62), as shown by the increase of $E_{g}$ (from iteration 50 to 61 ), and with only a moderate increase of $E_{i}$. For the remaining iterations, $\gamma$ is set back to its previous value: the interpenetration ratio slowly decreases while $E_{g}$ and $E_{d}$ remains constant. The use of polygonal cross-sections eventually permits to obtain a model which both maximizes $E_{g}$ and $E_{d}$ while maintaining a minimal interpenetration ratio, i.e. the final model is optimal with respect to our definition of $E$. It's worth noting that in this work no explicit convergence criterion has been used; the process is simply stopped after a limited number of iterations when the evolution of $E_{i}$ is small enough.

\subsection{Sensitivity to input data}

The example discussed in the previous section was extracted from a $\mu \mathrm{CT}$ with a resolution of $10 \mu \mathrm{m} /$ voxel. Evidently, the quality of the result will depend on the resolution and the dynamic of the initial $\mu \mathrm{CT}$.

The resolution of the $\mu \mathrm{CT}$ should be sufficient to describe accurately the geometry of the yarns. For the kind of materials we are interested in, a resolution up to $25 \mu \mathrm{m} /$ voxel would be adequate. The gray levels of the yarns and of the rest of the material also need to be sufficiently separated to construct the binary mask by using a simple threshold. In the case of a textile preform, this is easily achieved as shown in Sec. 2.3. For woven composites, i.e. when the preform has been infiltrated with a matrix, it demands more attention to obtain a sufficient 

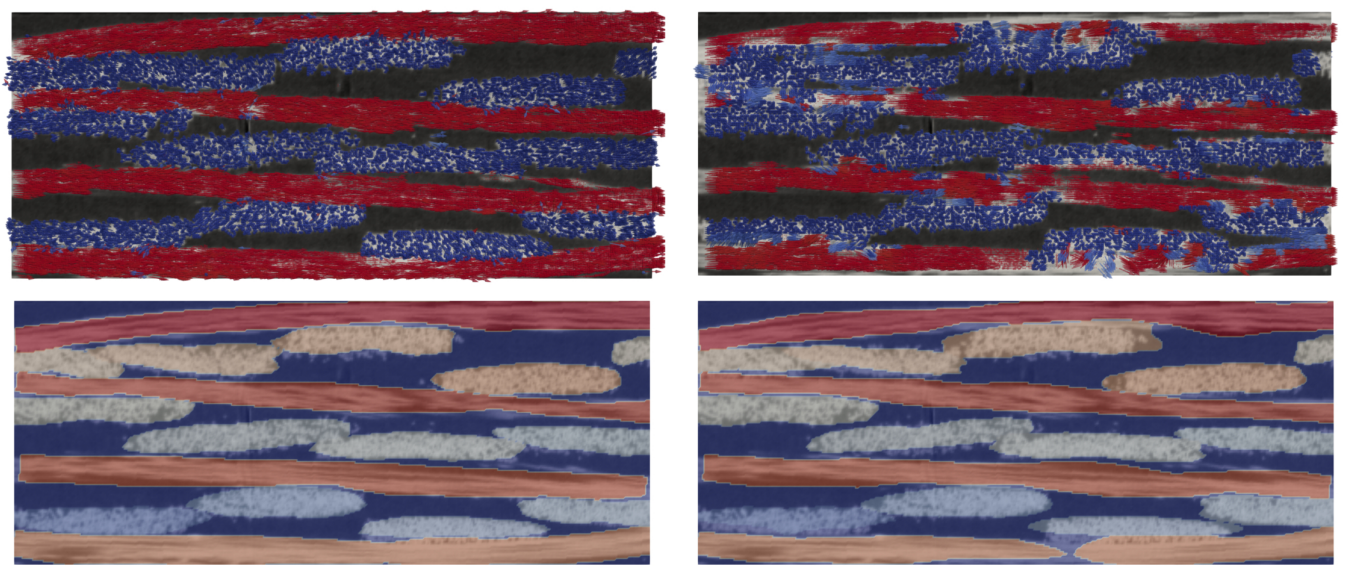

Figure 10: Influence of the $\mu \mathrm{CT}$ quality on the calculation of the local directions. Local directions are rendered as colored glyphs depending on their orientation (top row) for a full resolution $\mu \mathrm{CT}$ (left) and for the same $\mu \mathrm{CT}$ but with reduced quality (right). The corresponding models are shown in the bottom row.

contrast between the yarns and the matrix. This could be problematic when the $\mathrm{X}$-ray attenuation coefficients of the fibers and of the matrix are similar, e.g. in ceramic matrix composites. However, with well-tuned $\mu \mathrm{CT}$ acquisition parameters it is still possible to obtain the required contrast, as illustrated by the example in Sec. 5.4

The resolution and the dynamic of the $\mu \mathrm{CT}$ will also directly influence the calculation of the local directions. More precisely, the image should exhibit enough texture to allow the computation of the structure tensor (cf. Sec. 2.4). In the case of a textile fabric, the texture of the image is controlled by the fibers. However, it is not mandatory to fully resolved each individual fiber to retain enough texture. The structure tensor could be calculated as long as an appreciable gradient is present within the yarns. For woven fabric composites, intra-yarn porosity could suffice to create an acceptable texture. 
To better appreciate the sensitivity of our approach to the local directions, we have compared two segmentations that only differ in the quality of the local directions. The first one uses the local directions calculated with the fully resolved $\mu \mathrm{CT}(10 \mu \mathrm{m} / \mathrm{voxel})$. For the second one, we have downgraded the quality of the $\mu \mathrm{CT}$ by averaging the gray levels over a neighborhood of $4 \times 4 \times 4$ voxels. The 5 corresponding local directions are depicted in Fig. 10. It can be clearly seen that, in the latter case, the computed directions are noisier, especially where the wefts and warps touch each other, which result in erroneous estimation of the local directions. These discrepancies induce noticeable differences regarding the final geometry, as illustrated on Fig. 10, To minimize the incompatibilities between the wrongly estimated directions and those of the geometric model, the yarns are forced to locally reduce their cross-sections. It results in irregular cross-section variation along the yarn paths. In the case of unreliable local directions, this detrimental effect could be moderated by reducing the maximal value of $\beta$. The weight of the local directions will be minimized with respect to the other terms. Yet, the separation between weft and warp will solely rely on the penalization of the interpenetration. For this particular example, a resolution of at least 20 $\mu \mathrm{m} /$ voxel was necessary to obtain acceptable local directions.

\subsection{Comparison with other segmentation methods}

Our variational segmentation approach lead to optimized models that are visually satisfying and optimal with respect to the definition of the similarity we have introduced. This same measure could also be used to assess the quality of models obtained using other segmentation methods. It is thus possible to evaluate the average "metric difference" (as introduced by [32]) between the initial $\mu \mathrm{CT}$ and the various segmentations. In the following, we have restricted the compari- 

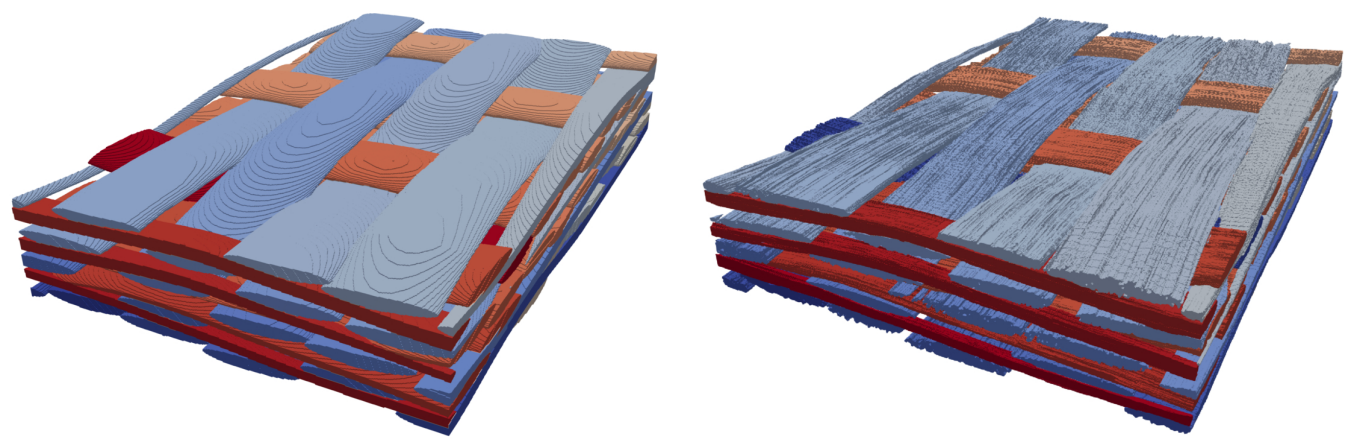

Figure 11: Resulting models for the $\mu \mathrm{CT}$ of Fig. 5 obtained using a geometric approach (left) and a manual segmentation (right).

son with two other models obtained respectively by considering constant elliptic cross-sections and by manual segmentation of the $\mu \mathrm{CT}$. The first model is similar to those that could be constructed with a priori geometric approaches. The constant elliptic cross-section is chosen as the average value of all the yarns within the $\mu \mathrm{CT}$. For the comparison to be fair, we haven't used the theoretical yarn paths 5 but the ones we have determined manually (see Sec. 2.2). The second model is obtained by a manual segmentation of the $\mu \mathrm{CT}$ using Avizo 9.0 (FEI): each yarn is contoured individually on 2D slices; these contours are then interpolated to generate the envelope. The corresponding models are illustrated on Fig. 11 and are to be compared the one of Fig. 5 .

However, a mere visual inspection is not sufficient to assess which model is the most similar to the $\mu \mathrm{CT}$. Thus, the similarity $E$ of each model is calculated following the procedure described in Sec. 3. The corresponding values of $E_{g}, E_{d}$, and $E_{i}$ are reported in Tab. 1 .

All three models achieve satisfying values of $E_{g}$ and $E_{d}$, the main difference 


\begin{tabular}{lccc}
\hline & $E_{g}$ & $E_{d}$ & $E_{i}$ \\
\hline Geometric model & $86.72 \%$ & $89.20 \%$ & $5.13 \%$ \\
Manual segmentation & $85.83 \%$ & $82.38 \%$ & $0.53 \%$ \\
Our approach & $86.90 \%$ & $90.20 \%$ & $0.01 \%$ \\
\hline
\end{tabular}

Table 1: Values of $E_{g}, E_{d}$ and $E_{i}$ for the models depicted in Fig 11 (geometric approach and manual segmentation) and for the model obtained with our approach (Fig. 5).

concerns the value of $E_{i}$. More precisely, the geometric model with constant elliptic cross-sections and the one generated using our approach exhibit almost identical values for $E_{g}$ and $E_{d}$. For this particular example, the shape of the crosssections are close to ellipses, and their size variation are limited: a approximate geometric model gives acceptable results regarding $E_{g}$ alone. The values of $E_{d}$ 5 are also almost equal since we have used the same prior yarn paths in both models. Yet, the geometric model leads to a much higher interpenetration ratio $(>5 \%$ vs. $<0.01 \%)$. This limitation is inherent in the use of elliptic cross-sections, as already discussed in Sec. 5.1. This geometric model corresponds to the first state of our model, i.e. before iteration 40 , when only elliptic cross-sections are used, and when penalization of the interpenetration is not yet taken into account. The small difference in $E_{i}$ (5\% for the geometric model vs. $2 \%$ to $4 \%$ before iteration 40 for our approach) could be related to the use of non-constant cross-sections in our model. It's worth recalling that a high interpenetration ratio could prevent the generation of a consistent finite element mesh of the preform, and therefore, must be avoided.

The manual segmentation could be considered as the most representative model with respect to the $\mu \mathrm{CT}$, even if it is dependant of the operator. Surprisingly, the 
manual segmentation has the lowest value of $E_{d}$ and $E_{g}$. A possible explanation is that the number of manual $2 \mathrm{D}$ contours along the yarn paths was not sufficient to capture accurately the variation of the cross-sections, as the envelope is constructed by linearly interpolating consecutive contours. The higher value of $E_{i}$ could be related to the segmentation process which is performed yarn by yarn: it is

5 thus sensitive to the choices of the operator when delimiting the frontier between yarns touching each other, especially when working on 2D slices only.

\subsection{Capability of our approach}

As illustrated above, our approach allows generating realistic and consistent models that are at least as good as manual segmentation, but also virtually free of interpenetration. The optimization of the geometry is also automatic and objective, which drastically reduces the manual processing time and eliminates the dependency on the operator's choices.

The preform used so far to discuss the implementation details and the convergence was intentionally simple, and may not fully demonstrate the capabilities of our approach. They are better illustrated by the following examples: the first one is an infiltrated oxide/oxide woven composite; the second one is extracted from a woven CMC junction, as depicted on Fig. 12 .

The first example demonstrates the possibility to successfully apply our approach to composites, as long as one can differentiate the yarns from the matrix. As discussed previously, the contrast can be enhanced by selecting appropriate $\mu \mathrm{CT}$ acquisition parameters. An alternative approach to deal with insufficient contrast could be to replace the binary mask with a probability map. By fitting the gray level histogram with a Gaussian mixture model, one can attribute a probability to belong either to yarns or to matrix to each voxel. The potential of our 

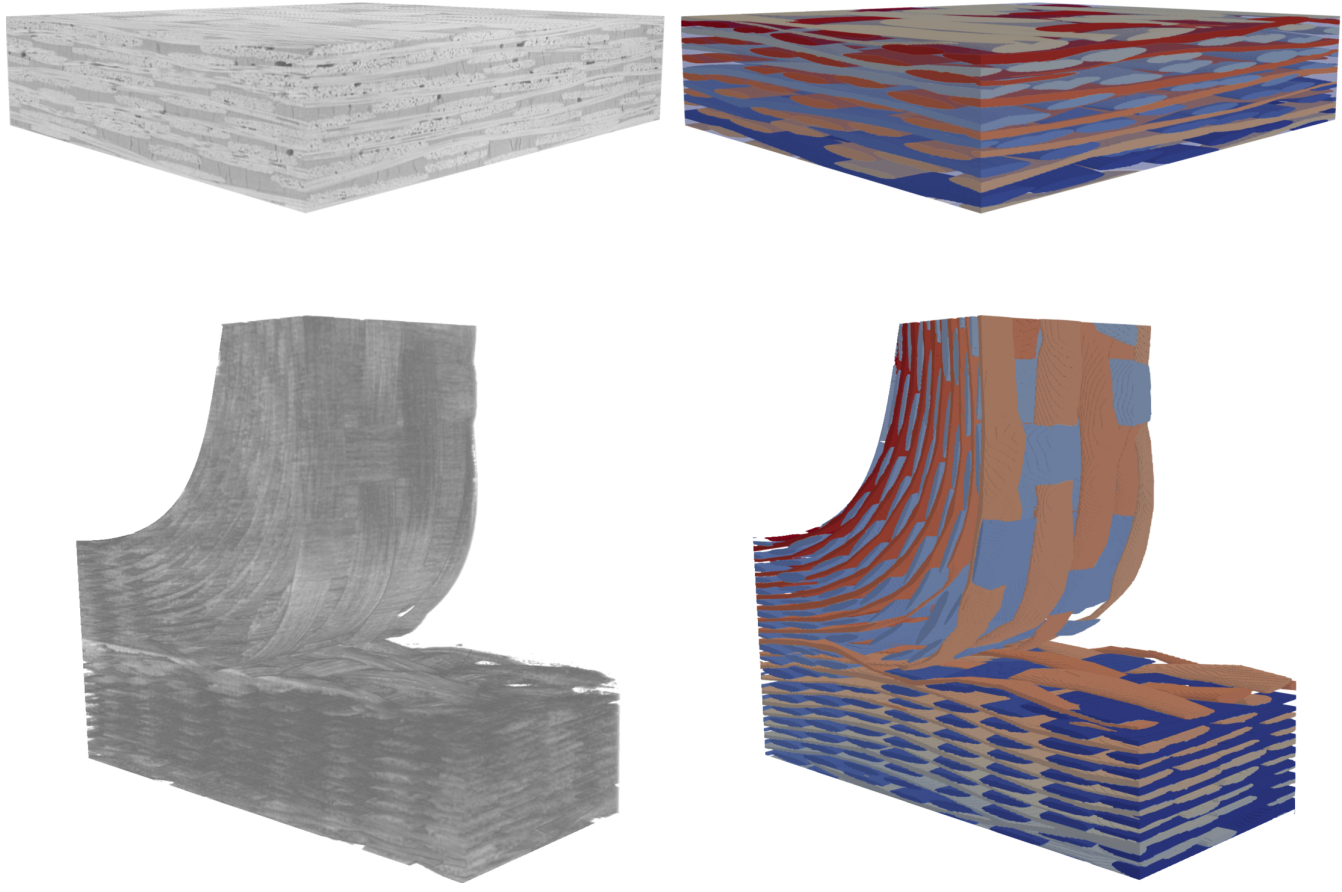

Figure 12: Illustration of the capabilities of our appraoch. $\mu \mathrm{CT}$ (left) and corresponding segmented model (right) for an infiltrated oxide/oxide composite (top) and for a fully woven CMC junction (bottom). 

junction [10]. Also, because of the size of the model, a complete manual segmentation of this part would be tedious and would require several days of manual processing.

\section{Conclusion and outlooks}

A variational segmentation approach has been proposed to tackle the challenging task of constructing realistic models of woven fabrics from $\mu \mathrm{CT}$. This approach is based on both data extracted from $\mu \mathrm{CT}$, and a prior geometric model. the yarns and inherently capture the variability of real materials. The concurrent use of a geometric model permits to handle a description of the yarns consistent with mesoscopic scale analysis, i.e. where each yarn is described individually. It also ensures that the prescribed weaving pattern is preserved. Our approach maximizes the resemblance between an initial geometric model and the $\mu \mathrm{CT}$ by iteratively improving a similarity measure thanks to a global-local heuristic optimization procedure. The similarity measure we introduced could be used to systematically evaluate the fidelity of geometric models with respect to a given 
$\mu \mathrm{CT}$; the metric difference between two models could also be computed using this same measure.

The inherent variabilities of real woven fabrics are also naturally captured by our approach. Therefore, the segmentation results could be used to generate statistics about the shape of the yarns, and their relation to the weaving pattern. It could 5 be of prime interest to better assess the interplay between the weaving process and the compaction of the yarns. These geometric informations could also help to detect weaving imperfections, or even missing yarns, by comparing our yarns segmentation with a reference idealized model. The output geometric models are topologically correct, and almost free of interpenetrations, which will greatly ease their conversion to FE meshes for further numerical simulations. These models will provide reference numerical models as they are a high-fidelity replica of the real material. These numerical twins would be of paramount importance, for example, to fully analyze results of in situ tests, and to better understand the link between the woven architecture and the damage initiation in CMC, see e.g. [30].

Our approach is almost fully automatic and versatile enough to tackle complex woven fabrics and composites: this allows to consider large $\mathrm{CMC}$ woven structural parts that couldn't be addressed by other methods. Yet, our approach still necessitates a preprocessing step to generate the prior geometric model. Even if the required manual intervention could be significant (up to a few hours for the largest part in Fig. 12), it remains much more tractable than for alternative approaches, as only the center lines of the yarns are needed. A solution to fully automatize our approach would be to initialize the prior model with the one of a geometric approach. The requirements are that the center lines are sufficiently close to the real ones for our approach to converge. This could be envisaged for 
simple weaves, but not yet for complex fabrics, at least from our point of view. The geometric models of complex textile structures (see e.g. [31]) appear to be too idealized to be used in our approach, and would necessitate further developments to include some stochastic considerations [21]. We have also shown that a minimal $\mu \mathrm{CT}$ resolution is required for the input data to be consistent with our

5 approach. It could be difficult to maintain a sufficient quality of the $\mu \mathrm{CT}$ when dealing with larger and more complex CMC parts. Experimental challenges will undoubtedly arise to conciliate acceptable resolution and quality with structural parts.

The ability to generate realistic FE models of CMC parts at the mesoscopic scale opens exciting perspectives for the simulation of such materials. But more detailed models will also necessarily demand more computational power and adequate numerical approaches. If full mesoscopic scale simulation is tempting, one can ask whether it is desirable to include such an amount of microstructural details to obtain satisfying results. Therefore, it would be interesting to evaluate the impact of the level of description of the microstructural details on the properties of such structures. The idea would be to find a compromise between the level of description and the computational burden for a given quantity of interest. This work is currently under investigation for woven composites regarding elastic properties and damage initiation.

\section{Acknowledgments}

This work was supported under PRC MECACOMP, French research project co-funded by DGAC and Safran group, managed by Safran group and involving Safran group, ONERA and CNRS. Thomas Revel and Thomas Vandellos from 
Safran Ceramics, and Véronique Brion from Safran Tech are acknowledged for fruitful discussions.

\section{References}

[1] A. Mouritz, M. Bannister, P. Falzon, K. Leong, Review of applications for advanced three-dimensional fibre textile composites, Composites Part A: Applied Science and Manufacturing 30 (12) (1999) 1445-1461 (1999).

[2] J. Whitcomb, Three-dimensional stress analysis of plain weave composites, in: Composite Materials: Fatigue and Fracture (Third Volume), ASTM International, 1991, pp. 417-417-22 (1991). doi:10.1520/stp17730s.

[3] F. Robitaille, A. Long, I. Jones, C. Rudd, Automatically generated geometric descriptions of textile and composite unit cells, Composites Part A: Applied Science and Manufacturing 34 (4) (2003) 303-312 (2003). doi:10.1016/ s1359-835x (03) 00063-0.

[4] S. Lomov, G. Perie, D. Ivanov, I. Verpoest, D. Marsal, Modeling threedimensional fabrics and three-dimensional reinforced composites: challenges and solutions, Textile Research Journal 81 (1) (2010) 28-41 (2010). doi:10.1177/0040517510385169.

[5] B. N. Cox, H. A. Bale, M. Begley, M. Blacklock, B.-C. Do, T. Fast, M. Naderi, M. Novak, V. P. Rajan, R. G. Rinaldi, et al., Stochastic virtual tests for high-temperature ceramic matrix composites, Annual Review of Materials Research 44 (1) (2014) 479-529 (2014). doi:10.1146/ annurev-matsci-122013-025024. 
[6] T. Gereke, C. Cherif, A review of numerical models for $3 \mathrm{~d}$ woven composite reinforcements, Composite Structures 209 (2019) 60-66 (2019). doi:10. $1016 /$ j.compstruct. 2018.10 .085 .

[7] S. Lomov, A. Gusakov, G. Huysmans, A. Prodromou, I. Verpoest, Textile geometry preprocessor for meso-mechanical models of woven composites, Composites Science and Technology 60 (11) (2000) 2083-2095 (2000). doi:10.1016/s0266-3538(00)00121-4.

[8] M. Sherburn, Geometric and mechanical modelling of textiles, Ph.D. thesis, University of Nottingham (2007). doi:10.1109/gmai .2007.21.

[9] G. Hivet, P. Boisse, Consistent 3d geometrical model of fabric elementary cell. application to a meshing preprocessor for $3 \mathrm{~d}$ finite element analysis, Finite Elements in Analysis and Design 42 (1) (2005) 25-49 (2005). doi : 10.1016/j.finel.2005.05.001.

[10] S. Yan, X. Zeng, L. Brown, A. Long, Geometric modeling of 3d woven preforms in composite t-joints, Textile Research Journal 88 (16) (2018) 18621875 (2018). doi:10.1177/0040517517712098.

[11] G. Grail, M. Hirsekorn, A. Wendling, G. Hivet, R. Hambli, Consistent finite element mesh generation for meso-scale modeling of textile composites with preformed and compacted reinforcements, Composites Part A: Applied Science and Manufacturing 55 (2013) 143-151 (2013). doi: 10.1016/j.compositesa.2013.09.001.

[12] Y. Wang, X. Sun, Digital-element simulation of textile processes, Com- 
posites Science and Technology 61 (2) (2001) 311-319 (2001). doi: $10.1016 / \mathrm{s} 0266-3538(00) 00223-2$

[13] D. Durville, Simulation of the mechanical behaviour of woven fabrics at the scale of fibers, International Journal of Material Forming 3 (S2) (2010) 1241-1251 (2010). doi:10.1007/s12289-009-0674-7.

[14] L. Huang, Y. Wang, Y. Miao, D. Swenson, Y. Ma, C.-F. Yen, Dynamic relaxation approach with periodic boundary conditions in determining the 3-d woven textile micro-geometry, Composite Structures 106 (2013) 417-425 (2013). doi:10.1016/j.compstruct.2013.05.057

[15] G. Couégnat, H. Ayadi, C. Saurat, E. Rohmer, Towards realistic geometric modeling of woven fabrics, in: 19th International Conference on Composite Materials (ICCM19), Montréal, Canada, 2013 (2013).

[16] A. Drach, B. Drach, I. Tsukrov, Processing of fiber architecture data for finite element modeling of 3d woven composites, Advances in Engineering Software 72 (2014) 18-27 (2014). doi:10.1016/j.advengsoft.2013. 06.006

[17] S. Green, A. Long, B. El Said, S. Hallett, Numerical modelling of 3d woven preform deformations, Composite Structures 108 (2014) 747-756 (2014). doi:10.1016/j.compstruct.2013.10.015.

[18] Z. Yousaf, P. Potluri, P. Withers, D. Mollenhauer, E. Zhou, S. Duning, Digital element simulation of aligned tows during compaction validated by computed tomography (ct), International Journal of Solids and Structures (2017). doi:10.1016/j.ijsolstr.2017.05.044. 
[19] R. G. Rinaldi, M. Blacklock, H. Bale, M. R. Begley, B. N. Cox, Generating virtual textile composite specimens using statistical data from microcomputed tomography: $3 \mathrm{~d}$ tow representations, Journal of the Mechanics and Physics of Solids 60 (8) (2012) 1561-1581 (2012). doi:10.1016/j. jmps.2012.02.008.

[20] H. Bale, M. Blacklock, M. R. Begley, D. B. Marshall, B. N. Cox, R. O. Ritchie, Characterizing three-dimensional textile ceramic composites using synchrotron x-ray micro-computed-tomography, Journal of the American Ceramic Society 95 (1) (2011) 392-402 (2011). doi:10.1111/j. $1551-2916.2011 .04802 \cdot \mathrm{x}$.

[21] A. Vanaerschot, B. N. Cox, S. V. Lomov, D. Vandepitte, Experimentally validated stochastic geometry description for textile composite reinforcements, Composites Science and Technology 122 (2016) 122-129 (2016). doi:10.1016/j.compscitech.2015.11.023.

[22] A. Wendling, J. L. Daniel, G. Hivet, E. Vidal-Sallé, P. Boisse, Meshing preprocessor for the mesoscopic $3 \mathrm{~d}$ finite element simulation of $2 \mathrm{~d}$ and interlock fabric deformation, Applied Composite Materials 22 (6) (2015) 869-886 (2015). doi:10.1007/s10443-015-9441-8.

[23] A. Vanaerschot, B. N. Cox, S. V. Lomov, D. Vandepitte, Stochastic multiscale modelling of textile composites based on internal geometry variability, Computers \& Structures 122 (2013) 55-64 (2013). doi:10.1016/j. compstruc.2012.10.026.

[24] H. H. Nagel, A. Gehrke, Spatiotemporally adaptive estimation and segmen- 
tation of of-fields, in: European Conference on Computer Vision, Springer, Springer Berlin Heidelberg, 1998, pp. 86-102 (1998). doi:10.1007/ bfb0054735.

[25] T. Brox, J. Weickert, B. Burgeth, P. Mrázek, Nonlinear structure tensors, in: J. Weickert, H. Hagen (Eds.), Visualization and processing of tensor fields, Vol. 24, Elsevier BV, 2006, pp. 41-55 (2006). doi:10.1016/j.imavis. 2005.09.010

[26] N. Naouar, E. Vidal-Sallé, J. Schneider, E. Maire, P. Boisse, Meso-scale fe analyses of textile composite reinforcement deformation based on x-ray computed tomography, Composite Structures 116 (2014) 165-176 (2014). doi:10.1016/j.compstruct.2014.04.026.

[27] I. Straumit, S. V. Lomov, M. Wevers, Quantification of the internal structure and automatic generation of voxel models of textile composites from x-ray computed tomography data, Composites Part A: Applied Science and Manufacturing 69 (2015) 150-158 (2015). doi:10.1016/j.compositesa. 2014.11 .016

[28] N. Naouar, E. Vidal-Salle, J. Schneider, E. Maire, P. Boisse, 3d composite reinforcement meso f.e. analyses based on x-ray computed tomography, Composite Structures 132 (2015) 1094-1104 (2015). doi:10.1016/j. compstruct.2015.07.005

[29] C. Chapoullié, J.-P. Da Costa, M. Cataldi, G. L. Vignoles, C. Germain, Orientation-guided two-scale approach for the segmentation and quantitative description of woven bundles of fibers from three-dimensional tomo- 
graphic images, Journal of Electronic Imaging 24 (6) (2015) 061113 (2015). doi:10.1117/1.jei.24.6.061113.

[30] V. Mazars, O. Caty, G. Couégnat, A. Bouterf, S. Roux, S. Denneulin, J. Pailhès, G. L. Vignoles, Damage investigation and modeling of 3d woven ceramic matrix composites from x-ray tomography in-situ tensile tests, Acta Materialia 140 (2017) 130-139 (2017). doi:10.1016/j.actamat. 2017.08 .034

[31] B. N. Cox, G. Nilakantan, O. Sudre, D. B. Marshall, Generating virtual specimens for complex non-periodic woven structures by converting machine instructions into topological ordering rules, Composite Structures 141 (2016) 63-78 (2016). doi:10.1016/j. compstruct.2016.01.009.

[32] A. Mendoza, J. Schneider, E. Parra, E. Obert, S. Roux, Differentiating 3d textile composites: A novel field of application for digital volume correlation, Composite Structures 208 (2019) 735-743 (2019). doi:10.1016/j. compstruct.2018.10.008

[33] J. Schindelin, I. Arganda-Carreras, E. Frise, V. Kaynig, M. Longair, T. Pietzsch, S. Preibisch, C. Rueden, S. Saalfeld, B. Schmid, et al., Fiji: an opensource platform for biological-image analysis, Nature Methods 9 (7) (2012) 676-682 (2012). doi:10.1038/nmeth. 2019

[34] C. Mulat, M. Donias, P. Baylou, G. Vignoles, C. Germain, Optimal orientation estimators for detection of cylindrical objects, Signal, Image and Video Processing 2 (1) (2007) 51-58 (2007). doi:10.1007/ s11760-007-0035-2 
[35] A. A. Taha, A. Hanbury, Metrics for evaluating 3d medical image segmentation: analysis, selection, and tool, BMC Medical Imaging 15 (1) (2015). doi:10.1186/s12880-015-0068-x.

[36] E. Potter, S. Pinho, P. Robinson, L. Iannucci, A. McMillan, Mesh generation and geometrical modelling of $3 \mathrm{~d}$ woven composites with variable tow crosssections, Computational Materials Science 51 (1) (2012) 103-111 (2012). doi:10.1016/j.commatsci.2011.06.034. 